\title{
Intermittent Sampled Data Control for Time-Varying Formation-Containment of the Multiagent System with/without Time Delay
}

\author{
Ming Chi, ${ }^{1,2}$ Xu-Long Wang, ${ }^{1,2}$ Yangming Dou, ${ }^{1,2}$ and Zhi-Wei Liu $\mathbb{D}^{1,2}$ \\ ${ }^{1}$ School of Artificial Intelligence and Automation, Huazhong University of Science and Technology, Wuhan 430074, China \\ ${ }^{2}$ Key Laboratory of Image Processing and Intelligent Control, Ministry of Education, \\ Huazhong University of Science and Technology, Wuhan 430074, China
}

Correspondence should be addressed to Zhi-Wei Liu; zwliu@hust.edu.cn

Received 12 March 2021; Accepted 30 April 2021; Published 7 May 2021

Academic Editor: Xiao Ling Wang

Copyright (c) 2021 Ming Chi et al. This is an open access article distributed under the Creative Commons Attribution License, which permits unrestricted use, distribution, and reproduction in any medium, provided the original work is properly cited.

Time-varying formation-containment problems for a second-order multiagent system (SOMAS) are studied via pulse-modulated intermittent control (PMIC) in this paper. A distributed control framework utilizing the neighbors' positions and velocities is designed so that leaders in the multiagent system form a formation, and followers move to the convex hull formed by each leader. Different from the traditional formation-containment problems, this paper applies the PMIC framework, which is more common and more in line with the actual control scenarios. Based on the knowledge of matrix theory, algebraic graph theory, and stability theory, some sufficient conditions are given for the time-varying formation-containment problem of the second-order multiagent system. Some numerical simulations are proposed to verify the effectiveness of the results presented in this paper.

\section{Introduction}

Many scholars start to pay attention to the multiagent systems (MASs) with the rapid development of complex network system theory. In recent decades, many major breakthroughs have been made in this field [1], and those results are also widely used in various fields of production and life, such as UAV cruise system [2], smart grid [3, 4], economic dispatching [5], and multiple underactuated surface vessels [6]. The most studied collaboration problems of MASs include swarm, consensus, formation, and distributed optimization. In very recent years, the clustering behavior of MASs has also attracted widespread interest, including but not limited to consensus [7-9], tracking, and formation [10].

In these cooperative control problems, many important advances have been made in the field of formation control and containment control. Huang et al. [11] studied the containment control problem of MASs via intermittent control-based sampled data information. Wang et al. [12] investigated the containment control problem of first-order MASs in the noisy communication environments. Rahimi et al. [13] studied time-varying formation control of collaborative heterogeneous MASs. However, most of the existing works are carried out on formation control and containment control separately. In many applications, both these cooperative behaviors often require simultaneous implementation. For example, in the coordination of multiple tanker airplanes and multiple UAVs, the tanker airplanes will form a specific formation in advance and wait for the UAVs to reach the area they surround. In order to solve this kind of problem, Dong et al. designed a continuous control strategy [14] for second-order MASs with multiple leaders and multiple followers, which can ensure the formation control of the leaders and containment of the followers. It is worth noting that the above work [14] adopted a continuous-time control which may be difficult to implement in some cases due to sampled measurement. The traditional zero-order sampling control adopts the same amplitude control input in the whole sampling interval, 
which can also lead to application difficulties. For instance, in a driverless system, it is difficult for the sensors on the vehicle to work all the time, allowing for fuel economy and other reasons.

Aiming at solving the aforementioned problems, the formation-containment problem of the second-order multiagent system (SOMAS) is studied in this paper, and the control framework of pulse-modulated intermittent control (PMIC) is adopted. The main contributions are given as follows. (i) The formation and the containment are achieved simultaneously in second-order MASs, where the leaders form a formation and the followers are contained in this formation. (ii) The PIMC is a framework that can unify impulsive control $[8,15]$ and zero-order sampling control [16]. It can be applied to a wider range of real-world scenarios. (iii) In this paper, some sufficient conditions are given for the parameters of the control strategy under with/ without time delay cases.

The remainder sections of this paper are described as follows. Section 2 lists the basic preliminary knowledge and a model of the problem to be studied. Section 3 introduces the formation-containment analysis without time delay. Section 4 obtains and provides the results when MASs contain time delay. Section 5 gives several simulations to verify the theorems are correct. Section 6 draws the conclusion.

\section{Preliminaries}

2.1. Graph Theory and Some Lemmas. Let $G=(V, E, A)$ be a weighted digraph, where $V=\{1, \ldots, N\}$ is a vertex set, $E \subseteq V \times V$ is a link set, and $A=\left[a_{i j}\right] \in \mathbb{R}^{N \times N}$ is a nonnegative weighted adjacency matrix. The information flow from vertex $j$ to vertex $i$ is represented by a directed link $e_{i j}=(j, i)$. The elements of matrix $A$ are described as follows: $a_{i j}>0$ if $e_{i j} \in E$, and $a_{i j}=0$, otherwise. Furthermore, $a_{i i}=0$ for all $i \in V$, and $a_{i j}=a_{j i}$ in an undirected topology. Denote $N_{i}=\left\{v_{j} \in V:\left(v_{j}, v_{i}\right) \in E\right\}$ as the set of neighbors of node $v_{i}$. The Laplacian matrix $L$ is described in terms of $L=D-A$, where $D=\operatorname{diag}\left\{D_{1}, D_{2}, \ldots, D_{N}\right\}$ is the in-degree matrix with $D_{i}=\sum_{j=1}^{N} a_{i j}$. A sequence of edges with different nodes of $V$ as $\left\{\left(v_{i}, v_{i 1}\right),\left(v_{i}, v_{i 2}\right), \ldots,\left(v_{i}, v_{j}\right)\right\}$ can be obtained if there is a directed path from the $i$ th agent to the jth agent.

In a MAS, a SOMAS with $N$ agents is considered, and there are $M$ followers and $N-M$ leaders. Assume that followers can receive messages from the leaders or followers, while the leaders can only receive messages from the leaders. Let $F=\{1,2, \ldots, M\}$ and $E=\{M+1, M+2, \ldots, N\}$ denote the sets of followers and leaders, respectively. And the Laplacian matrix $L_{N}$ is described as

$$
L_{N}=\left[\begin{array}{cc}
L_{1} & L_{2} \\
0 & L_{3}
\end{array}\right]
$$

where $L_{1} \in \mathbb{R}^{M \times M}, L_{2} \in \mathbb{R}^{M \times(N-M)}$, and $L_{3} \in \mathbb{R}(N-M) \times$ $(N-M)$.
Lemma 1 (see [17]). A complex characteristic polynomial $R(z)=z^{2}+s z+r$ is Hurwitz stable if and only if $\operatorname{Re}(s)>0$ and $\operatorname{Re}(s) \operatorname{Im}(s) \operatorname{Im}(r)+\operatorname{Re}^{2}(s) \operatorname{Re}(r)-\operatorname{Im}^{2}(r)>0$.

Lemma 2 (see [18]). If directed graph $G$ contains a spanning tree, then the Laplacian matrix $L \in \mathbb{R}^{N \times N}$ of $G$ has a simple zero eigenvalue with $\overrightarrow{1}_{N}$ as the associated eigenvector, and all the other $N-1$ eigenvalues have positive real parts.

Assumption 1. Each follower of graph $G$ has at least one directed path from one leader.

Assumption 2. Let $G_{E}$ be the graph associated with the leaders in the MAS, and $G_{E}$ contains a spanning tree.

Lemma 3 (see [19]). By Assumptions 1 and 2, it is obtained that the eigenvalues of $L_{1}$ have positive real parts, each row of $-L_{1}^{-1} L_{2}$ has a sum equal, and each entry of $-L_{1}^{-1} L_{2}$ is nonnegative.

2.2. Model Formulation and Some Definitions. The control input of the $i$ th agent is denoted by $u_{i} \in \mathbb{R}^{n}$, position by $p_{i} \in \mathbb{R}^{n}$, and velocity by $v_{i} \in \mathbb{R}^{n}$, respectively. Consider a SOMAS as

$$
\begin{aligned}
& \dot{p}_{i}(t)=v_{i}(t), \\
& \dot{v}_{i}(t)=u_{i}(t) .
\end{aligned}
$$

In the following, for the sake of description, we assume that $n=1$. However, more cases such as $n>1$ can be derived by using the Kronecker product.

Let, $B=\left[\begin{array}{l}0 \\ 1\end{array}\right], \quad$ and $\quad x_{i}(t)=\left[p_{i}(t), v_{i}(t)\right]^{T}(i=1,2$, $\ldots, N)$. Then, the dynamic equation of the $i$ th agent can be described in a neat form as follows:

$$
\dot{x}_{i}(t)=A x_{i}(t)+B u_{i}(t) .
$$

Definition 1. With the bounded initial state of each agent being chosen arbitrarily, the leaders in MAS (3) are said to realize time-varying formation if there exists a vector function $c(t) \in \mathbb{R}^{2}$ such that

$$
\lim _{t \longrightarrow \infty}\left(x_{i}(t)-h_{i}(t)-c(t)\right)=0 \quad(i \in E),
$$

where $h_{i}(t)=\left[h_{i p}(t), h_{i v}(t)\right]^{T}$ is a piecewise continuously differentiable vector. Then, we can denote the time-varying formation as $h_{E}(t)=\left[h_{M+1}^{T}(t), h_{M+2}^{T}(t), \ldots, h_{N}^{T}(t)\right]^{T} \in$ $\mathbb{R}^{2(N-M)}$.

Definition 2. Similarly, with the bounded initial state of each agent being chosen arbitrarily, MAS (3) is said to achieve containment if there exist nonnegative constants $\tau_{k, j}(k \in F, j \in E)$ such that $\sum_{j=M+1}^{N} \tau_{k, j}=1$ and 


$$
\lim _{t \longrightarrow \infty}\left(x_{k}(t)-\sum_{j=M+1}^{N} \tau_{k, j} x_{j}(t)\right)=0 .
$$

\section{Formation-Containment Analysis without Time Delay}

In this section, we mainly study how to design the PMIC protocol to make MAS (3) achieve the time-varying formation-containment and propose some sufficient and necessary conditions for parameters. We will investigate the time-varying formation-containment problem in two steps. The first step is to transform the formation-containment problem into a stability problem. The second step is to solve the stability problem according to the related theory.

3.1. Problem Transformation. Consider the following PMIC protocols:

$$
\begin{aligned}
u_{i}(t)= & a\left(t-t_{k}\right) K_{1} \sum_{j \in N_{i}} a_{i j}\left(\left(x_{i}\left(t_{k}\right)-h_{i}\left(t_{k}\right)\right)\right. \\
& \left.-\left(x_{j}\left(t_{k}\right)-h_{j}\left(t_{k}\right)\right)\right), \quad i \in E, \\
u_{i}(t)= & a\left(t-t_{k}\right) K_{2} \sum_{j \in N_{i}} a_{i j}\left(x_{i}\left(t_{k}\right)-x_{j}\left(t_{k}\right)\right), \quad i \in F,
\end{aligned}
$$

where $K_{1}=\left[k_{11}, k_{12}\right] \in \mathbb{R}^{1 \times 2}$ and $K_{2}=\left[k_{21}, k_{22}\right] \in \mathbb{R}^{1 \times 2}$ are feedback gain matrices and $t_{k}<t<t_{k+1}$. The pulse function $a(t)$ is described as

$$
a(t)= \begin{cases}\widehat{a}(t), & t \in(0, d], \\ 0, & t \notin(0, d],\end{cases}
$$

where $\widehat{a}(t)$ is a piecewise continuous function. Let $T=t_{k+1}-$ $t_{k}$ and $d<T$ be the control duration within a complete sampling cycle. $\left(t_{k}+d, t_{k+1}\right)$ is the rest interval, and $\left(t_{k}, t_{k}+\right.$ $d]$ is the control interval [20].

Under the control framework (6), MAS (3) can be described in a compact form as follows:

$$
\begin{aligned}
\dot{x}_{E}(t)= & \left(I_{N-M} \otimes A\right) x_{E}(t)+a\left(t-t_{k}\right)\left(\left(L_{3} \otimes B K_{1}\right) x_{E}\left(t_{k}\right)\right. \\
& \left.-\left(L_{3} \otimes B K_{1}\right) h_{E}\left(t_{k}\right)\right), \\
\dot{x}_{F}(t)= & \left(I_{M} \otimes A\right) x_{F}(t)+a\left(t-t_{k}\right)\left(\left(L_{1} \otimes B K_{2}\right) x_{F}\left(t_{k}\right)\right. \\
& \left.+\left(L_{2} \otimes B K_{2}\right) x_{E}\left(t_{k}\right)\right),
\end{aligned}
$$

where $x_{E}(t)=\left[x_{M+1}^{T}(t), x_{M+2}^{T}(t), \ldots, x_{N}^{T}(t)\right]^{T}$ and $x_{F}(t)=$ $\left[x_{1}^{T}(t), x_{2}^{T}(t), \ldots, x_{M}^{T}(t)\right]^{T}$.

Let $\theta_{i}(t)=x_{i}(t)-h_{i}(t)(i \in E)$ and $\theta_{E}(t)=\left[\theta_{M+1}^{T}(t)\right.$, $\left.\theta_{M+2}^{T}(t), \ldots, \theta_{N}^{T}(t)\right]^{T}$. Then, system (8) can be written as

$$
\begin{aligned}
\dot{\theta}_{E}(t)= & \left(I_{N-M} \otimes A\right) \theta_{E}(t)+a\left(t-t_{k}\right)\left(L_{3} \otimes B K_{1}\right) \theta_{E}\left(t_{k}\right) \\
& +\left(I_{N-M} \otimes A\right) h_{E}(t)-\left(I_{N-M} \otimes I_{2}\right) \dot{h}_{E}(t) .
\end{aligned}
$$

The eigenvalues of $L_{3}$ relating to $G_{E}$ are denoted by $\lambda_{i}(i \in E)$, where $\lambda_{M+1}=0$ with the eigenvector as
$p_{M+1}=\left(\mathbf{1}_{N-M} / \sqrt{N-M}\right), \quad$ and $\quad 0<\operatorname{Re}\left(\lambda_{M+2}\right) \leq \cdots \leq$ $\operatorname{Re}\left(\lambda_{N}\right)$. Let $P_{E}^{-1} L_{3} P_{E}=J_{E}$, where $J_{E}$ is the Jordan canonical form of $L_{3}, \quad P_{E}=\left[p_{M+1}, p_{M+2}, \ldots, p_{N}\right], \quad$ and $P_{E}^{-1}=\left[\tilde{p}_{M+1}, \tilde{p}_{M+2}, \ldots, \widetilde{p}_{N}\right]^{H}$. By Lemma 2, one has $J_{E}=\operatorname{diag}\left\{0, \bar{J}_{E}\right\}$, where $\bar{J}_{E}$ consists of the Jordan blocks relating to $\lambda_{i}(i=M+2, M+3, \ldots, N)$. Let $\widetilde{\theta}_{E}(t)=\left(P_{E}^{-1} \otimes I_{2}\right) \theta_{E}(t)$; then, system (9) can be rewritten as

$$
\begin{aligned}
\tilde{\theta}_{E}(t)= & \left(I_{N-M} \otimes A\right) \tilde{\theta}_{E}(t)+a\left(t-t_{k}\right)\left(P_{E}^{-1} L_{3} P_{E} \otimes B K_{1}\right) \tilde{\theta}_{E}\left(t_{k}\right) \\
& +\left(P_{E}^{-1} \otimes A\right) h_{E}(t)-\left(P_{E}^{-1} \otimes I_{2}\right) \dot{h}_{E}(t) .
\end{aligned}
$$

Let

$$
\widetilde{P}_{E}=\left[\widetilde{p}_{M+2}, \widetilde{p}_{M+3}, \ldots, \widetilde{p}_{N}\right]^{H},
$$

$\alpha_{E}(t)=\left(\widetilde{p}_{M+1}^{H} \otimes I_{2}\right) \theta_{E}(t), \quad$ and $\beta_{E}(t)=\left(\widetilde{P}_{E} \otimes I_{2}\right) \theta_{E}(t)$. Then, system (10) can be divided as

$$
\begin{aligned}
\dot{\alpha}_{E}(t)= & A \alpha_{E}(t)+\left(\widetilde{p}_{M+1}^{H} \otimes A\right) h_{E}(t)-\left(\widetilde{p}_{M+1}^{H} \otimes I_{2}\right) \dot{h}_{E}(t), \\
\dot{\beta}_{E}(t)= & \left(I_{N-M-1} \otimes A\right) \beta_{E}(t)+a\left(t-t_{k}\right)\left(\bar{J}_{E} \otimes B K_{1}\right) \beta_{E}\left(t_{k}\right) \\
& +\left(\widetilde{P}_{E} \otimes A\right) h_{E}(t)-\left(\widetilde{P}_{E} \otimes I_{2}\right) \dot{h}_{E}(t) .
\end{aligned}
$$

Let $\quad \delta_{i}=\sum_{j \in N_{i}} w_{i j}\left(x_{i}(t)-x_{j}(t)\right), i \in F, \quad$ and $\delta_{F}(t)=\left[\delta_{1}(t), \delta_{2}(t), \ldots, \delta_{M}(t)\right]$. Then, the following lemma is used to transform the formation-containment problem.

Lemma 4. MAS (3) under the PMIC framework (6) can achieve time-varying formation-containment if

$$
\left\{\begin{array}{l}
\lim _{t \longrightarrow \infty} \beta_{E}(t)=0_{2(N-M-1)}, \\
\lim _{t \longrightarrow \infty} \delta_{F}(t)=0_{2 M} .
\end{array}\right.
$$

Proof. It is able to be proved by a similar way in [14].

Remark 1. Lemma 4 is proved because the following results in [14] are worked out:

$$
\begin{aligned}
\lim _{t \longrightarrow \infty}\left(x_{E}(t)-h_{E}(t)-\left(\frac{\mathbf{1}_{N-M}}{\sqrt{N-M}} \otimes I_{2}\right) \alpha_{E}(t)\right) & =0_{2(N-M)}, \\
\lim _{t \longrightarrow \infty}\left(x_{F}(t)-\left(-L_{1}^{-1} L_{2} \otimes I_{2}\right) x_{E}(t)\right) & =0_{2 M} .
\end{aligned}
$$

Considering Lemma 3, we can conclude that equation (13) satisfies Definitions 1 and2, respectively. In other words, Lemma 4 can be proved by the above two equations.

3.2. Control Design. By means of Lemma 4, the time-varying formation-containment problem can be transformed into the convergence analysis of $\beta_{E}(t)$ and $\delta_{F}(t)$. This section presents the conditions for the parameters in control protocol (6) when $\beta_{E}(t)$ and $\delta_{F}(t)$ converge to 0 .

Assumption 3. For $\forall i \in\{M+1, M+2, \ldots, N\}$, 
$\lim _{t \longrightarrow \infty}\left(A\left(h_{i}(t)-h_{j}(t)\right)-\left(\dot{h}_{i}(t)-\dot{h}_{j}(t)\right)\right)=0, \quad j \in N_{i}$.

Lemma 5. Let

$$
\dot{\tilde{\beta}}_{E}(t)=\left(I_{N-M-1} \otimes A\right) \widetilde{\beta}_{E}(t)+a\left(t-t_{k}\right)\left(\bar{J}_{E} \otimes B K_{1}\right) \widetilde{\beta}_{E}\left(t_{k}\right) .
$$

Under Assumption 3, $\lim _{t \longrightarrow \infty} \beta_{E}(t)=0_{2(N-M-1)}$ is satisfied if

$$
\lim _{t \rightarrow \infty} \tilde{\beta}_{E}(t)=0_{2(N-M-1)} .
$$

Proof. If Assumption 3 holds, one has

$$
\lim _{t \longrightarrow \infty}\left(\left(L_{3} \otimes A\right) h_{E}(t)-\left(L_{3} \otimes I_{2}\right) \dot{h}_{E}(t)\right)=0 .
$$

Substitute $L_{3}=P_{E} J_{E} P_{E}^{-1}$ into (17), and then premultiply both sides of (17) by $P_{E}^{-1} \otimes I_{2}$. One can obtain that

$$
\lim _{t \longrightarrow \infty}\left(\left(\bar{J}_{E} \widetilde{P}_{E} \otimes A\right) h_{E}(t)-\left(\bar{J}_{E} \widetilde{P} \otimes I_{2}\right) \dot{h}_{E}(t)\right)=0 .
$$

Under Assumption 2 and Lemma 2, $\bar{J}_{E}$ is nonsingular, obviously. By premultiplying both sides of (18) by $\bar{J}_{E}^{-P} \otimes I_{2}$, we have

$$
\lim _{t \longrightarrow \infty}\left(\left(\widetilde{P}_{E} \otimes A\right) h_{E}(t)-\left(\widetilde{P} \otimes I_{2}\right) \dot{h}_{E}(t)\right)=0 .
$$

Considering equation (19), Lemma 5 is set up.

Lemma 6. $\forall i \in\{M+2, M+3, \ldots, N\}$, equation (16) holds if the following inequalities are satisfied:

$$
\begin{gathered}
\frac{k_{12}}{k_{11}}>\frac{d_{2}}{d_{1}}, \\
\left(\frac{2 k_{12}}{k_{11} T}-\frac{2 d_{2}}{d_{1} T}\right)^{2}\left(\frac{2 d_{2}}{d_{1} T}-\frac{2 k_{12}}{k_{11} T}-\frac{4 \operatorname{Re}\left(\lambda_{i}\right)}{k_{11} d_{1}\left|\lambda_{i}\right|^{2} T}-1\right)-\frac{16 \operatorname{Im}^{2}\left(\lambda_{i}\right)}{k_{11}^{2} d_{1}^{2}\left|\lambda_{i}\right|^{4} T^{2}}>0,
\end{gathered}
$$

where $d_{1}=\int_{0}^{d} \widehat{a}(s) d s$ and $d_{2}=\int_{0}^{d} s \hat{a}(s) d s$.

Proof. Equation (16) holds if and only if $\widetilde{\beta}_{E}$ in (15) is asymptotically stable. The solution of (15) can be written as

$$
\tilde{\beta}_{E}(t)=e^{C\left(t-t_{0}\right)} \tilde{\beta}_{E}\left(t_{0}\right)+e^{C t} \int_{t_{0}}^{t} e^{-C s} a\left(s-t_{k}\right) D \tilde{\beta}_{E}\left(t_{k}\right) \mathrm{d} s,
$$

where $C=I_{N-M-1} \otimes A$ and $D=\bar{J}_{E} \otimes B K_{1}$. Let $t_{k}=t_{0}$ and $t_{k}+d=t$ in (22); then, we have

$$
\begin{aligned}
\tilde{\beta}_{E}\left(t_{k}+d\right)= & e^{C d} \widetilde{\beta}_{E}\left(t_{k}\right)+e^{C\left(t_{k}+d\right)} \int_{t_{k}}^{t_{k}+d} e^{-C s} a\left(s-t_{k}\right) \\
& D \widetilde{\beta}_{E}\left(t_{k}\right) \mathrm{d} s .
\end{aligned}
$$

According to $C^{2}=0 a$ and the theory of matrix function, one can obtain that

$$
\tilde{\beta}_{E}\left(t_{k}+d\right)=\left(I_{2(N-M-1)}+d C+d_{1} D+\left(d d_{1}-d_{2}\right) C D\right) \tilde{\beta}_{E}\left(t_{k}\right) .
$$

Notice that $\widetilde{\beta}_{E}\left(t_{k+1}\right)=\widetilde{\beta}_{E}\left(t_{k}+T\right)$; then, the system can be discretely expressed as

$$
\tilde{\beta}_{E}\left(t_{k+1}\right)=\left(I_{2(N-M-1)}+T C+d_{1} D+\left(T d_{1}-d_{2}\right) C D\right) \tilde{\beta}_{E}\left(t_{k}\right) .
$$

Let $\quad \Gamma=I_{2(N-M-1)}+T C+d_{1} D+\left(T d_{1}-d_{2}\right) C D$ and $\operatorname{det}\left(s I_{2(N-M-1)}-\Gamma\right)=0$. It yields

$$
s^{2}-\left[2+k_{12} d_{1} \lambda_{i}+k_{11} \lambda_{i}\left(T d_{1}-d_{2}\right)\right] s+1+k_{12} d_{1} \lambda_{i}-k_{11} d_{2} \lambda_{i}=0 .
$$

Considering $\lambda_{i} \neq 0$, then it is focused on the conditions that the eigenvalues of $\Gamma$ are encircled by the unit circle. By applying a bilinear transformation, $z=((s+1) /(s-1))$, an updated polynomial can be found as

$$
\begin{aligned}
R(z)= & z^{2}+\frac{2\left(k_{12} d_{1} \lambda_{i}-k_{11} \lambda_{i} d_{2}\right)}{k_{11} \lambda_{i} d_{1} T} z \\
& +\frac{2 k_{11} \lambda_{i} d_{2}-2 k_{12} d_{1} \lambda_{i}-k_{11} d_{1} \lambda_{i} T-4}{k_{11} \lambda_{i} d_{1} T} .
\end{aligned}
$$

$\|s\|<1$ in (26) can be obtained if and only if $R(z)$ in (27) is Hurwitz stable. Based on Lemma 1, if (27) is stable, then both equations (20) and (21) hold.

Lemma 7. $\lim _{t \longrightarrow \infty} \delta_{F}(t)=0_{2 M}$ holds if and only if $\forall i \in\{1,2, \ldots, M\}$,

$$
\begin{gathered}
\frac{k_{22}}{k_{21}}>\frac{d_{2}}{d_{1}} \\
\left(\frac{2 k_{22}}{k_{21} T}-\frac{2 d_{2}}{d_{1} T}\right)^{2}\left(\frac{2 d_{2}}{d_{1} T}-\frac{2 k_{22}}{k_{21} T}-\frac{4 \operatorname{Re}\left(\lambda_{i}\right)}{k_{21} d_{1}\left|\lambda_{i}\right|^{2} T}-1\right)-\frac{\left.16 \operatorname{Im}^{2}\left(\lambda_{i}\right)\right)}{k_{21}^{2} d_{1}^{2}\left|\lambda_{i}\right|^{4} T^{2}}>0,
\end{gathered}
$$

where $d_{1}=\int_{0}^{d} \widehat{a}(s) d s$ and $d_{2}=\int_{0}^{d} s \widehat{a}(s) d s$.

Proof. It is obtained that

$$
\delta_{F}(t)=\left(L_{2} \otimes I_{2}\right) x_{E}(t)+\left(L_{1} \otimes I_{2}\right) x_{F}(t) .
$$

Substituting (8) into (30), we can obtain that

$$
\begin{gathered}
\dot{\delta}_{F}(t)=\left(I_{M} \otimes A\right) \delta_{F}(t)+a\left(t-t_{k}\right)\left(L_{1} \otimes B K_{2}\right) \delta_{F}\left(t_{k}\right) \\
a\left(t-t_{k}\right)\left(L_{2} L_{3} \otimes B K_{1}\right)\left(x_{E}\left(t_{k}\right)-h_{E}\left(t_{k}\right)\right) .
\end{gathered}
$$

By Lemmas 4 and 5, MAS (8) can achieve formation $h_{E}(t)$. Considering Definition 1, when the leaders' formation $h_{E}(t)$ is achieved, then 


$$
\begin{array}{r}
\lim _{t \longrightarrow \infty}\left(L_{2} L_{3} \otimes B K_{1}\right)\left(x_{E}\left(t_{k}\right)-h_{E}\left(t_{k}\right)\right) \\
=\lim _{t \longrightarrow \infty}\left(L_{2} L_{3} \otimes B K_{1}\right)\left(\mathbf{1} \otimes c\left(t_{k}\right)\right) .
\end{array}
$$

Since $L_{3} 1=0$, one has

$$
\lim _{t \longrightarrow \infty}\left(L_{2} L_{3} \otimes B K_{1}\right)\left(x_{E}\left(t_{k}\right)-h_{E}\left(t_{k}\right)\right)=0 .
$$

Then, consider the following system:

$$
\dot{\delta}_{F}(t)=\left(I_{M} \otimes A\right) \delta_{F}(t)+a\left(t-t_{k}\right)\left(L_{1} \otimes B K_{2}\right) \delta_{F}\left(t_{k}\right) .
$$

$\lim _{t \longrightarrow \infty} \delta_{F}(t)=0_{2 M}$ means that system (34) is asymptotically stable. Similar to the analysis of Lemma 5 , the conditions that make system (34) asymptotically stable are worked out with (28) and (29).

Theorem 1. MAS (3) under Assumption 3 and PMIC framework (6) realizes formation-containment if the following conditions simultaneously hold:

(i) $\forall i \in\{1,2, \ldots, M\}$,

$$
\begin{array}{r}
\frac{k_{22}}{k_{21}}>\frac{d_{2}}{d_{1}}, \\
\left(\frac{2 k_{22}}{k_{21} T}-\frac{2 d_{2}}{d_{1} T}\right)^{2}\left(\frac{2 d_{2}}{d_{1} T}-\frac{2 k_{22}}{k_{21} T}-\frac{4 \operatorname{Re}\left(\lambda_{i}\right)}{k_{21} d_{1}\left|\lambda_{i}\right|^{2} T}-1\right)-\frac{16 \operatorname{Im}^{2}\left(\lambda_{i}\right)}{k_{21}^{2} d_{1}^{2}\left|\lambda_{i}\right|^{4} T^{2}}>0 .
\end{array}
$$

(ii) $\forall i \in\{M+2, M+3, \ldots, N\}$,

$$
\begin{array}{r}
\frac{k_{12}}{k_{11}}>\frac{d_{2}}{d_{1}}, \\
\left(\frac{2 k_{12}}{k_{11} T}-\frac{2 d_{2}}{d_{1} T}\right)^{2}\left(\frac{2 d_{2}}{d_{1} T}-\frac{2 k_{12}}{k_{11} T}-\frac{4 \operatorname{Re}\left(\lambda_{i}\right)}{k_{11} d_{1}\left|\lambda_{i}\right|^{2} T}-1\right)-\frac{16 \operatorname{Im}^{2}\left(\lambda_{i}\right)}{k_{11}^{2} d_{1}^{2}\left|\lambda_{i}\right|^{4} T^{2}}>0,
\end{array}
$$

where $d_{1}=\int_{0}^{d} \widehat{a}(s) d s$ and $d_{2}=\int_{0}^{d} s \widehat{a}(s) d s$.

Proof. It is proved naturally by Lemmas 4, 6, and 7 .

Remark 2. When selecting parameters in the control rules, we generally give $K_{1}, K_{2}, d_{1}$, and $d_{2}$ that meet the conditions first. According to the values of these parameters and conditions (35) and (36) in Theorem 1, we can solve parameter $T$ as follows:

(i) $\forall i \in\{1,2, \ldots, M\}$,

$$
T<\min \frac{M_{1}^{3}\left|\lambda_{i}\right|^{4}+4 \operatorname{Re}\left(\lambda_{i}\right)\left|\lambda_{i}\right|^{2} M_{1}^{2}}{16 k_{21} d_{1} \operatorname{Im}^{2}\left(\lambda_{i}\right)+k_{21} d_{1} M_{1}^{2}\left|\lambda_{i}\right|^{4}},
$$

where $M_{1}=2 k_{21} d_{2}-2 k_{22} d_{1}$. (ii) $\forall i \in\{M+2, M+3, \ldots, N\}$,

$$
T<\min \frac{M_{1}^{3}\left|\lambda_{i}\right|^{4}+4 \operatorname{Re}\left(\lambda_{i}\right)\left|\lambda_{i}\right|^{2} M_{1}^{2}}{16 k_{11} d_{1} \operatorname{Im}^{2}\left(\lambda_{i}\right)+k_{11} d_{1} M_{1}^{2}\left|\lambda_{i}\right|^{4}},
$$

where $M_{2}=2 k_{11} d_{2}-2 k_{12} d_{1}$.

\section{Formation-Containment Analysis with Time Delay}

We know that the time delay cannot be ignored due to its widely existence in the real world. Then, a control protocol with considering time delay is proposed as follows:

$$
\begin{aligned}
& u_{i}(t)=a\left(t-t_{k}\right) K_{1} \sum_{j \in N_{i}} w_{i j}\left(\left(x_{i}\left(t_{k}-\tau\right)-h_{i}\left(t_{k}\right)\right)-\left(x_{j}\left(t_{k}-\tau\right)-h_{j}\left(t_{k}\right)\right)\right), \quad i \in E, \\
& u_{i}(t)=a\left(t-t_{k}\right) K_{2} \sum_{j \in N_{i}} w_{i j}\left(x_{i}\left(t_{k}-\tau\right)-x_{j}\left(t_{k}-\tau\right)\right), \quad i \in F .
\end{aligned}
$$


The control protocol (39) is substituted into MAS (3), and we can obtain

$$
\begin{aligned}
& \dot{x}_{E}(t)=\left(I_{N-M} \otimes A\right) x_{E}(t)+a\left(t-t_{k}\right)\left(\left(L_{3} \otimes B K_{1}\right) x_{E}\left(t_{k}-\tau\right)-\left(L_{3} \otimes B K_{1}\right) h_{E}\left(t_{k}\right)\right), \\
& \dot{x}_{F}(t)=\left(I_{M} \otimes A\right) x_{F}(t)+a\left(t-t_{k}\right)\left(\left(L_{1} \otimes B K_{2}\right) x_{F}\left(t_{k}-\tau\right)+\left(L_{2} \otimes B K_{2}\right) x_{E}\left(t_{k}-\tau\right)\right) .
\end{aligned}
$$

Considering Assumption 3, when there is a time delay in the system, similar to Section 3, we have

$$
\begin{aligned}
& \dot{\tilde{\beta}}_{E}(t)=\left(I_{N-M-1} \otimes A\right) \tilde{\beta}_{E}(t)+a\left(t-t_{k}\right)\left(\bar{J}_{E} \otimes B K_{1}\right) \tilde{\beta}_{E}\left(t_{k}-\tau\right), \\
& \dot{\delta}_{F}(t)=\left(I_{M} \otimes A\right) \delta_{F}(t)+a\left(t-t_{k}\right)\left(L_{1} \otimes B K_{2}\right) \delta_{F}\left(t_{k}-\tau\right) .
\end{aligned}
$$

It is not hard to see that Lemma 4 also applies to cases with time delay. Therefore, we still transform the formationcontainment problem of the SOMAS into the stability problem of the system.

Remark 3. Considering that the time delay of the actual system is not long and the intermittent control has the

advantage of selectively adjusting the control time interval $d$, we assume that the time delay $\tau<\min \{d, T-d\}$.

Lemma 8. $\lim _{t \rightarrow \infty} \widetilde{\beta}_{E}(t)=0_{2(N-M-1)}$ holds with time delay if $\forall i \in\{M+2, M+3, \ldots, N\}$,

$$
-\frac{2 \tau}{T}-\frac{2 d_{2}}{d_{1} T}+\frac{2 k_{12}}{k_{11} T}>0
$$

$$
\left(-\frac{2 \tau}{T}-\frac{2 d_{2}}{d_{1} T}+\frac{2 k_{12}}{k_{11} T}\right)^{2}\left[-\frac{4 \operatorname{Re}\left(\lambda_{i}\right)}{\left|\lambda_{i}\right|^{2} k_{11} d_{1} T}-1+\frac{2 \tau}{T}+\frac{2 d_{2}}{d_{1} T}-\frac{2 k_{12}}{k_{11} T}\right]-\frac{16 \operatorname{Im}^{2}\left(\lambda_{i}\right)}{\left|\lambda_{i}\right|^{4} k_{11}^{2} d_{1}^{2} T^{2}}>0,
$$

where $d_{1}=\int_{0}^{d} \widehat{a}(s) d s, d_{2}=\int_{0}^{d} s \hat{a}(s) d s$, and $\tau$ is the time delay.

Proof. Similarly as Lemma 6, differential equation (41) is solved. Since $\tau<\min \{d, T-d\}$, when $t \in\left[t_{k}, t_{k+1}\right)$, we can obtain that

$$
\widetilde{\beta}_{i}(t)=M\left(t-t_{k}\right) \widetilde{\beta}_{i}\left(t_{k}\right)+N\left(t-t_{k}\right) \widetilde{\beta}_{i}\left(t_{k}-\tau\right)
$$

where $\quad M(\chi)=\left[\begin{array}{ll}1 & t \\ 0 & 1\end{array}\right] \quad$ and $\quad N(\chi)$ $=\left[\begin{array}{cc}\left(t d_{1}-d_{2}\right) \lambda_{i} k_{11} & \left(t d_{1}-d_{2}\right) \lambda_{i} k_{12} \\ \lambda_{i} k_{11} d_{1} & \lambda_{i} k_{12} d_{1}\end{array}\right]$. To solve the stability problem, we need to consider the iterative problem of the system. When $t=t_{k}$, one has

$$
\begin{aligned}
\tilde{\beta}_{i}\left(t_{k}\right) & =M(T) \tilde{\beta}_{i}\left(t_{k-1}\right)+N(T) \widetilde{\beta}_{i}\left(t_{k-1}-\tau\right), \\
\tilde{\beta}_{i}\left(t_{k}-\tau\right) & =M(T-\tau) \tilde{\beta}_{i}\left(t_{k-1}\right)+N(T-\tau) \tilde{\beta}_{i}\left(t_{k-1}-\tau\right) .
\end{aligned}
$$

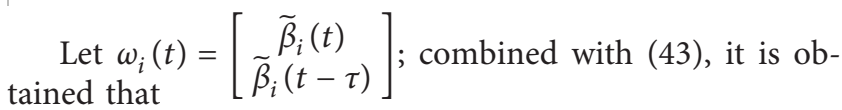

$$
\omega_{i}(t)=\left[\begin{array}{c}
\tilde{\beta}_{i}(t) \\
\tilde{\beta}_{i}(t-\tau)
\end{array}\right]=\Gamma_{1} \Gamma_{2}^{k}\left[\begin{array}{c}
\tilde{\beta}_{i}\left(t_{0}\right) \\
\tilde{\beta}_{i}\left(t_{0}-\tau\right)
\end{array}\right]
$$

where $\quad \Gamma_{1}=\left[\begin{array}{cc}M\left(t-t_{k}\right) & N\left(t-t_{k}\right) \\ M\left(t-t_{k}-\tau\right) & N\left(t-t_{k}-\tau\right)\end{array}\right] \quad$ and $\Gamma_{2}=\left[\begin{array}{cc}M(T) & N(T) \\ M(T-\tau) & N(T-\tau)\end{array}\right]$. It is easy to get that $\omega_{i}(t) \longrightarrow 0$ if the eigenvalues of $\Gamma_{2}$ meet $\|s\|<1$. Let $\left|s I_{4}-\Gamma_{2}\right|=0$. It yields

$$
s^{4}+a_{1} s^{3}+a_{0} s^{2}=0
$$

where $\quad a_{1}=-\lambda_{i} k_{11} d_{1} T+\lambda_{i} k_{11} d_{1} \tau+\lambda_{i} k_{11} d_{2}-\lambda_{i} k_{12} d_{1}-2$ and $a_{0}=-\lambda_{i} k_{11} d_{1} \tau-\lambda_{i} k_{11} d_{2}+\lambda_{i} k_{12} d_{1}+1$. We can obtain that two eigenvalues of $\Gamma_{2}$ satisfy $s_{1}=s_{2}=0$. Then, the remaining proof process has been omitted, which is similar to the proof of Lemma 6 . 
Lemma 9. $\lim _{t \rightarrow \infty} \delta_{F}(t)=0_{2 M}$ holds with time delay if $\forall i \in\{1,2, \ldots, M\}$,

$$
\begin{array}{r}
-\frac{2 \tau}{T}-\frac{2 d_{2}}{d_{1} T}+\frac{2 k_{22}}{k_{21} T}>0, \\
\left(-\frac{2 \tau}{T}-\frac{2 d_{2}}{d_{1} T}+\frac{2 k_{22}}{k_{21} T}\right)^{2}\left[-\frac{4 \operatorname{Re}\left(\lambda_{i}\right)}{\left|\lambda_{i}\right|^{2} k_{21} d_{1} T}-1+\frac{2 \tau}{T}+\frac{2 d_{2}}{d_{1} T}-\frac{2 k_{22}}{k_{21} T}\right]-\frac{16 \operatorname{Im}^{2}\left(\lambda_{i}\right)}{\left|\lambda_{i}\right|^{4} k_{21}^{2} d_{1}^{2} T^{2}}>0,
\end{array}
$$

where $d_{1}=\int_{0}^{d} \widehat{a}(s) d s, d_{2}=\int_{0}^{d} s \hat{a}(s) d s$, and $\tau$ is the time delay.

Proof. This proof is analogous to Lemma 8 and is therefore omitted.

Theorem 2. MAS (3) under Assumption 3 and control framework (39) realizes formation-containment if conditions (42), (43), (48), and (49) hold.

Proof. It is not hard to prove by applying Lemmas 4,8 , and 9.

\section{Simulation}

In this section, a two-dimensional formation-containment case of the MAS will be shown by numerical simulations. The simulations illustrate the effectiveness of the results in this paper. In this two-dimensional formation-containment case, suppose there are three leaders and two followers in the MAS. The Laplace matrix of the directed topology is given as

$$
L=\left[\begin{array}{ccccc}
2 & -\frac{2}{3} & -\frac{1}{3} & -1 & 0 \\
-1 & 2 & 0 & 0 & -1 \\
0 & 0 & 1 & 0 & -1 \\
0 & 0 & -1 & 1 & 0 \\
0 & 0 & 0 & -1 & 1
\end{array}\right] .
$$

Note that $\quad p_{i}(t) \in \mathbb{R}^{2}, \quad v_{i}(t) \in \mathbb{R}^{2}, \quad$ and $x_{i}(t)=\left[p_{i X}(t), v_{i X}(t), p_{i Y}(t), v_{i Y}(t)\right]^{T}, \quad i \in\{1,2, \ldots, 5\}$. Then, matrices $A$ and $B$ in dynamic equation (3) should be extended as

$$
\begin{aligned}
& A=I_{2} \otimes\left[\begin{array}{ll}
0 & 1 \\
0 & 0
\end{array}\right], \\
& B=I_{2} \otimes\left[\begin{array}{l}
0 \\
1
\end{array}\right] .
\end{aligned}
$$

The formation function for leaders is given by

$$
h_{i}(t)=\left[\begin{array}{c}
\sin \left(t+\frac{2(i-3) \pi}{3}\right) \\
\cos \left(t+\frac{2(i-3) \pi}{3}\right) \\
\cos \left(t+\frac{2(i-3) \pi}{3}\right) \\
-\sin \left(t+\frac{2(i-3) \pi}{3}\right)
\end{array}\right] \quad(i=3,4,5) .
$$

The eigenvalues of $L$ are $\lambda_{1}=2.8165, \lambda_{2}=1.1835, \lambda_{3}=0$, $\lambda_{4}=1.5+0.866 i$, and $\lambda_{5}=1.5-0.866 i$. The function $\widehat{a}(t)=$ $(t / d)$ is set, and the control duration $d=0.2$ is chosen; then, $d_{1}=0.1$ and $d_{2}=0.01333$.

When we do not care about time delays, it follows from (20) and (28) that $k_{12} / k_{11}>0.1333$ and $k_{22} / k_{21}>0.1333$. The feedback gains are chosen as $K_{1}=I_{2} \otimes[-1,-2]$ and $K_{2}=I_{2} \otimes[-1,-2]$. According to inequalities (21) and (29), $T<1.5396$ is obtained, and $T=0.6$ is chosen. When time delays are taken into account, we assume the time delay $\tau=0.1$. According to Lemmas 8 and 9 , we also choose the feedback gains as $K_{1}=I_{2} \otimes[-1,-2]$ and $K_{2}=I_{2} \otimes[-1,-2]$ and set $T=0.6$.

The simulation results are shown in figures. Among them, Figures 1 and 2 are the trajectories of the position and velocity of each agent changing without considering the time delay, in which the initial state is marked with a circle and the final state is marked with a triangular row. Figures 3 and 4 show the trajectories when time delay is taken into account. The same initial state is indicated by a circle, and the final state is indicated by a triangular row. The initial states of all agents are randomly selected.

During the simulation, the five agents begin with arbitrary initial states. As time goes on, the three leaders reach a time-varying circular formation, and two followers move into the convex hull formed by leaders. At the same time, it can be seen that the system with time delay converges more slowly with the same sampling period. 


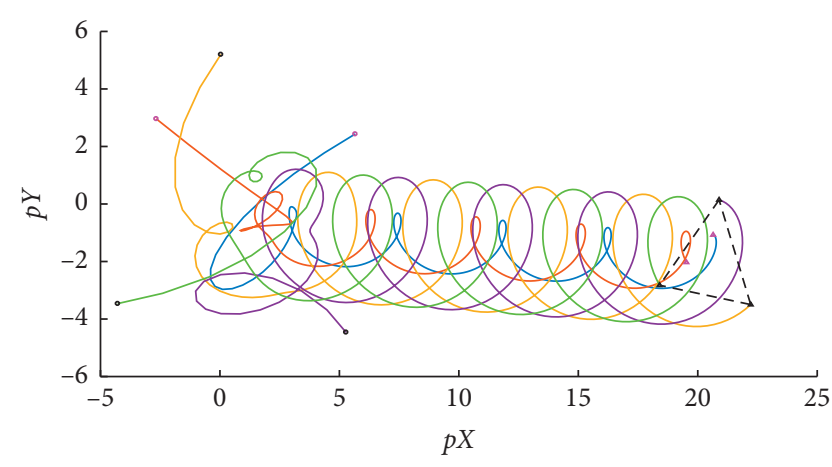

FIGURE 1: The trajectory of agents' positions over time without time delay.

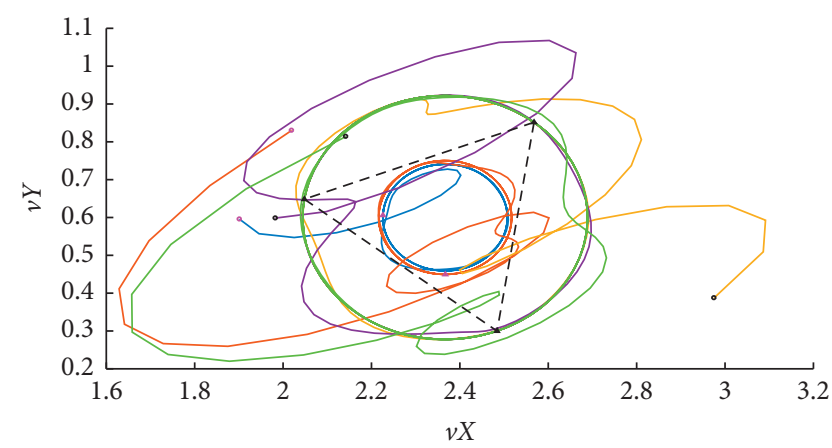

FIGURE 2: The trajectory of agents' velocities over time without time delay.

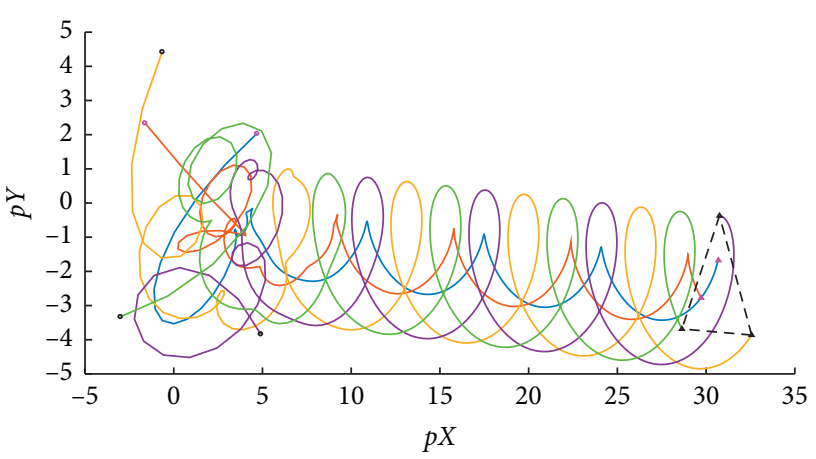

FIGURE 3: The trajectory of agents' positions over time with time delay.

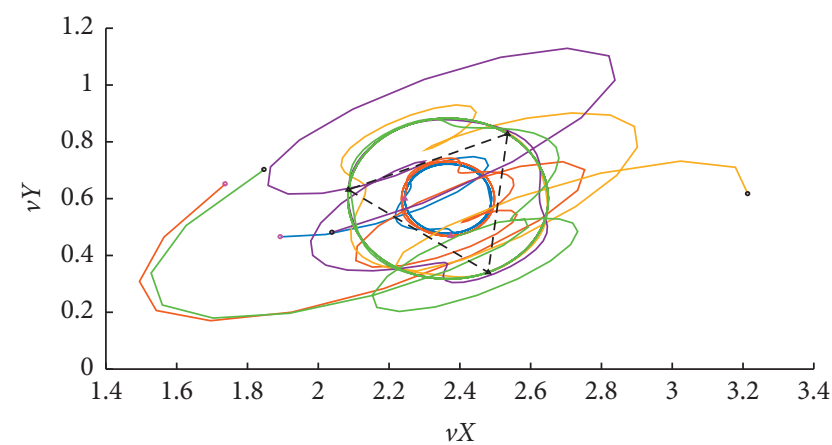

FIGURE 4: The trajectory of agents' velocities over time with time delay.

\section{Conclusion}

Time-varying formation-containment problems of the SOMAS were studied in this paper. Based on the fundamental theorems of graph theory and matrix theory, the formation-containment problem is transformed into the stability problem of the SOMAS. The PMIC protocols are designed, and the sufficient conditions for the time-varying formation-containment of the SOMAS are given by proving three theorems. The simulations show the correctness of the proposed theoretical results. It is expected that the future work will be carried out on the nonlinear MAS, and the conclusion of this paper will be applied to the actual multiintelligent vehicle experimental platform.

\section{Data Availability}

No data were used to support this study.

\section{Conflicts of Interest}

The authors declare that there are no conflicts of interest regarding the publication of this paper.

\section{Acknowledgments}

This work was supported by the National Natural Science Foundation of China (Grant nos. 61972170 and 61973133).

\section{References}

[1] H. Su, X. Wang, and Z. Lin, "Flocking of multi-agents with a virtual leader," IEEE Transactions on Automatic Control, vol. 54, no. 2, pp. 293-307, 2009.

[2] X. Dong, B. Yu, Z. Shi, and Y. Zhong, "Time-varying formation control for unmanned aerial vehicles: theories and applications," IEEE Transactions on Control Systems Technology, vol. 23, no. 1, pp. 340-348, 2014.

[3] X. Hu, Z.-W. Liu, G. Wen, X. Yu, and C. Li, "Branch-wise parallel successive algorithm for online voltage regulation in distribution networks," IEEE Transactions on Smart Grid, vol. 10, no. 6, pp. 6678-6689, 2019.

[4] X. Hu, Z.-W. Liu, G. Wen, X. Yu, and C. Liu, "Voltage control for distribution networks via coordinated regulation of active and reactive power of dgs," IEEE Transactions on Smart Grid, vol. 11, no. 5, pp. 4017-4031, 2020.

[5] X. Hu, H. Zhou, Z.-W. Liu, X. Yu, and C. Li, "Hierarchical distributed scheme for demand estimation and power reallocation in a future power grid," IEEE Transactions on Industrial Informatics, vol. 13, no. 5, pp. 2279-2290, 2017.

[6] Z.-W. Liu, H. Hou, and Y.-W. Wang, "Formation-containment control of multiple underactuated surface vessels with sampling communication via hierarchical sliding mode approach," ISA Transactions, 2019.

[7] M. Chi, X.-L. Wang, D.-X. He, and Z.-W. Liu, "Multiconsensus of second-order multiagent networks via pulse-modulated intermittent control," Complexity, vol. 2020, Article ID 1059026, 2020.

[8] Z.-W. Liu, G. Wen, X. Yu, Z.-H. Guan, and T. Huang, "Delayed impulsive control for consensus of multiagent systems with switching communication graphs," IEEE 
Transactions on Cybernetics, vol. 50, no. 7, pp. 3045-3055, 2020.

[9] H. Su, H. Wu, and J. Lam, "Positive edge-consensus for nodal networks via output feedback," IEEE Transactions on Automatic Control, vol. 64, no. 3, pp. 1244-1249, 2019.

[10] X. Dong, Y. Zhou, Z. Ren, and Y. Zhong, "Time-varying formation tracking for second-order multi-agent systems subjected to switching topologies with application to quadrotor formation flying," IEEE Transactions on Industrial Electronics, vol. 64, no. 6, pp. 5014-5024, 2017.

[11] N. Huang, Z. Duan, and G. Chen, "Some necessary and sufficient conditions for consensus of second-order multiagent systems with sampled position data," Automatica, vol. 63 , pp. $148-155,2016$.

[12] Y. Wang, L. Cheng, Z. G. Hou, M. Tan, and M. Wang, "Containment control of multi-agent systems in a noisy communication environment," Automatica, vol. 50, no. 7, pp. 1922-1928, 2014.

[13] R. Rahimi, F. Abdollahi, and K. Naqshi, "Time-varying formation control of a collaborative heterogeneous multi agent system," Robotics and Autonomous Systems, vol. 62, no. 12, pp. 1799-1805, 2014.

[14] L. Han, X. Dong, Q. Li, and Z. Ren, "Formation-containment control for second-order multi-agent systems with timevarying delays," Neurocomputing, vol. 218, pp. 439-447, 2016.

[15] Z.-W. Liu, Z.-H. Guan, X. Shen, and G. Feng, "Consensus of multi-agent networks with aperiodic sampled communication via impulsive algorithms using position-only measurements," IEEE Transactions on Automatic Control, vol. 57, no. 10, pp. 2639-2643, 2012.

[16] H. Su, Y. Liu, and Z. Zeng, "Second-order consensus for multiagent systems via intermittent sampled position data control," IEEE Transactions on Cybernetics, vol. 50, no. 5, pp. 2063-2072, 2020.

[17] P. C. Parks and V. Hahn, Stability Theory, Prentice-Hall, Inc., Hoboken, NJ, USA, 1993.

[18] W. Ren and R. W Beard, "Consensus seeking in multiagent systems under dynamically changing interaction topologies," IEEE Transactions on Automatic Control, vol. 50, no. 5, pp. 655-661, 2005.

[19] Z. Meng, W. Ren, and Z. You, "Distributed finite-time attitude containment control for multiple rigid bodies," Automatica, vol. 46, no. 12, pp. 2092-2099, 2010.

[20] Z.-W. Liu, X. Yu, Z.-H. Guan, B. Hu, and C. Li, "Pulsemodulated intermittent control in consensus of multiagent systems," IEEE Transactions on Systems, Man, and Cybernetics: Systems, vol. 47, no. 5, pp. 783-793, 2017. 